\title{
Taxonomy and phylogenetic position of Fimbristylis fusiformis, a new species of Cyperaceae from Thailand
}

\author{
K. Wangwasit ${ }^{1}$, A.M. Muasya ${ }^{2}$, P. Chantaranothai ${ }^{3}$, D.A. Simpson ${ }^{4}$
}

\section{Key words}

conservation status

Fimbristylis

phylogeny

taxonomy

\begin{abstract}
Fimbristylis fusiformis, an unusual new species of Cyperaceae from Thailand, is described and illustrated This taxon has a single terminal spikelet per culm with a semi-distichous glume arrangement, bisexual flowers that lack perianth parts, and pistil with persistent style whose base is slightly swollen and trigonous nutlets with pubescent ribs. Phylogenetic reconstruction using ITS sequence data places this taxon in Abildgaardieae and sister to the rest of Fimbristylis. The species has a conservation status of Least Concern (LC).
\end{abstract}

Published on 28 February 2017

\section{INTRODUCTION}

The genus Fimbristylis Vahl, comprising c. 300 species, occurs mostly in the tropics and subtropics with some species occurring in warm temperate regions (Govaerts et al. 2007). The genus is morphologically diverse, with inflorescences ranging from complex umbel-like structures to a solitary spikelet. The glumes are generally spirally arranged in ellipsoid spikelets, more rarely distichously arranged, the bisexual flowers lack perianth parts and their deciduous styles have distinctly thickened base, and the nutlets often have distinct surface patterns. Fimbristylis is currently (e.g., Govaerts et al. 2007) treated to include the segregate genus Abildgaardia Vahl, the latter only differing in having a distichous glume arrangement (Goetghebeur 1998). The genus was revised for Thailand by Simpson \& Koyama (1998), who enumerated 60 species for that country. A study of undetermined material by one of us (DAS) suggested a previously undescribed species was present in eastern Thailand (Map 1). Further herbarium studies, together with associated fieldwork, confirmed that the species was new. We inferred the phylogenetic position of this new species using DNA sequence data, and describe and illustrate it here (Fig. 1-3).

\section{MATERIALS AND METHODS}

Specimens were examined from herbaria at BK, BKF, K, KKU and QBG. Fieldwork was undertaken in September 2008 and November 2011. Observations of inflorescences and fruits were made with an Olympus SZ-PT binocular microscope. Mature nutlets from the spikelet of the holotype were collected for scanning electron microscopy. Selected material was mounted on stubs with double-sided adhesive tape and coated with gold using a SC7620 mini sputter-coater (Polaron range). Micro-

\footnotetext{
Department of Biology, Faculty of Science, Mahasarakham University, Maha Sarakham 44150, Thailand.

2 Department of Biological Sciences, University of Cape Town, Private Bag X3, Rondebosch 7701, South Africa.

${ }^{3}$ Applied Taxonomic Research Center, Department of Biology, Faculty of Science, Khon Kaen University, Khon Kaen 40002, Thailand.

${ }^{4}$ Royal Botanic Gardens, Kew, Richmond, Surrey TW9 3AB, UK; Department of Botany, School of Natural Sciences, Trinity College Dublin, Dublin 2, Ireland; corresponding author e-mail: D.Simpson@kew.org.
}

graphs were generated using a JSM6460LV scanning electron microscope (JEOL Ltd.)

Using a silica gel dried sample of the new taxon, DNA was extracted using the CTAB method and the ITS marker amplified and sequenced using standard protocols and primers (Muasya et al. 2014). Contigs of forward and reverse sequences were assembled using the STADEN package (Staden 1996). Additional ITS DNA sequences, primarily taken from studies of Abildgaardieae (Ghamkhar et al. 2007) and Cypereae (Yano et al. 2012), were downloaded from GenBank. The matrix thus assembled (Table 1) included a total of 90 taxa, with representatives of subfamily Cyperoideae, tribes Abildgaardieae, Cypereae, Eleocharideae, Fuireneae, Scirpeae with Cladium as outgroup. The matrix was aligned using Muscle (v. 3.8.31; Edgar 2004), and further manually aligned in BioEdit (v. 7.0.9; Hall 1999). The aligned matrix was analysed using maximum parsimony in PAUP* (Swofford 2002), with heuristic searches using the random-addition-sequence method with 10000 replicates, Tree-Bisection-Reconnection (TBR) branch-swapping with the Multrees option in effect and no maximum tree number set. Node support was evaluated using bootstrap analyses with 500 replicates, repeating the heuristic search procedure (with 10 replicates) above.

\section{RESULTS AND DISCUSSION}

Although the new species was easily identifiable as a member of subfamily Cyperoideae (absence of the spicoid floral structure (Simpson et al. 2003), individual flowers comprising stamens and gynoecium subtended by a glume), its generic placement within Cyperoideae was not immediately obvious. The combination of morphological characters, especially in the structure of the gynoecium, with the scabrous style, the apparent lack of a disjunction between the style base and nutlet, the fusiform shape of the nutlet and the fimbriate hairs at its apex and base (Fig. 1), suggested a genus other than Fimbristylis. Indeed, some aspects of the plant's gross morphology (e.g., a single, terminal spikelet) were superficially similar to genera such as Trichophorum (tribe Scirpeae) whereas the lack of perianth parts and possession of a persistent style with an indistinct base on the nutlet, were reminiscent of features observed in Isolepis (tribe Cypereae). 
Table 1 List of taxa sampled for the phylogenetic study.

\begin{tabular}{|c|c|c|c|}
\hline Tribe & Taxon & Voucher & Accession number \\
\hline Abildgaardieae & $\begin{array}{l}\text { Arthrostylis aphylla R.Br. } \\
\text { Bulbostylis barbata (Rottb.) C.B.Clarke } \\
\text { Bulbostylis densa (Wall.) Hand.-Mazz. } \\
\text { Bulbostylis funckii (Steud.) C.B.Clarke } \\
\text { Crosslandia setifolia W.Fitzg. } \\
\text { Fimbristylis aestivalis Vahl } \\
\text { Fimbristylis bisumbellata (Forssk.) Bubani } \\
\text { Fimbristylis complanata (Retz.) Link } \\
\text { Fimbristylis composita Latz } \\
\text { Fimbristylis composita Latz } \\
\text { Fimbristylis composita Latz } \\
\text { Fimbristylis cymosa R.Br. } \\
\text { Fimbristylis densa S.T.Blake } \\
\text { Fimbristylis dichotoma (L.) Vahl } \\
\text { Fimbristylis diphylloides Makino } \\
\text { Fimbristylis ferruginea (L.) Vahl } \\
\text { Fimbristylis fusiformis Wangwasit \& D.A.Simpson } \\
\text { Fimbristylis globulosa (Retz.) Kunth } \\
\text { Fimbristylis lanceolata C.B.Clarke } \\
\text { Fimbristylis laxiglumis Latz } \\
\text { Fimbristylis littoralis Gaudich. } \\
\text { Fimbristylis longispica Steud. } \\
\text { Fimbristylis miliacea (L.) Vahl } \\
\text { Fimbristylis neilsonii F.Muell. } \\
\text { Fimbristylis ovata (Burm.f.) J.Kern. } \\
\text { Fimbristylis oxystachya F.Muell. } \\
\text { Fimbristylis pachyptera S.T.Blake } \\
\text { Fimbristylis pauciflora R.Br. } \\
\text { Fimbristylis pierotii Miq. } \\
\text { Fimbristylis polytrichoides (Retz.) R.Br. } \\
\text { Fimbristylis rara R.Br. } \\
\text { Fimbristylis schultzii Boeckeler } \\
\text { Fimbristylis sericea (Poir.) R.Br. } \\
\text { Fimbristylis sieberiana Kunth } \\
\text { Fimbristylis sieboldii Miq. } \\
\text { Fimbristylis squarrosa Vahl } \\
\text { Fimbristylis stauntonii Debeaux \& Franch. } \\
\text { Fimbristylis subbispicata Nees } \\
\text { Fimbristylis tetragona R.Br. } \\
\text { Fimbristylis tristachya R.Br. } \\
\text { Fimbristylis vaginata (R.Br.) Domin } \\
\text { Fimbristylis velata R.Br. }\end{array}$ & 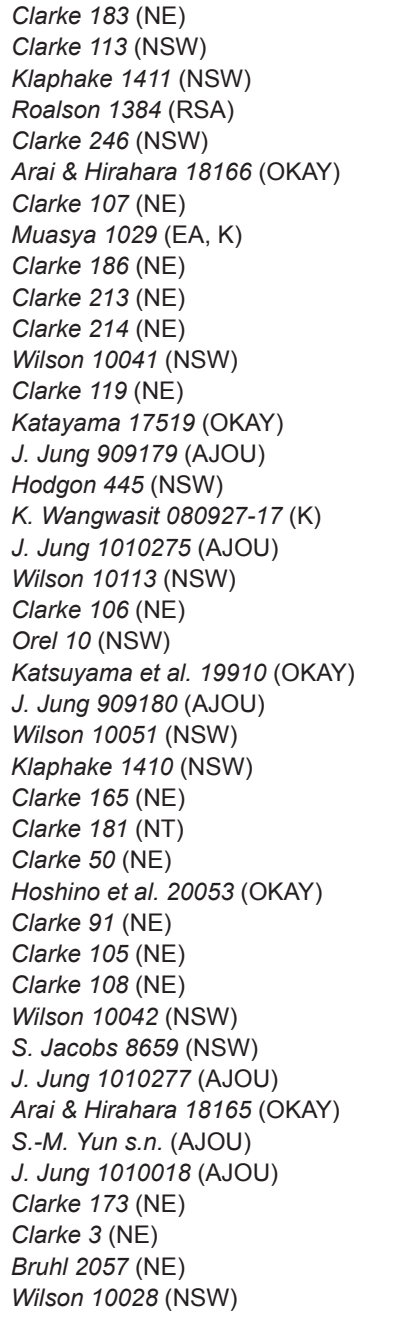 & $\begin{array}{l}\text { AY506757 } \\
\text { AY506764 } \\
\text { AY506763 } \\
\text { AF190616 } \\
\text { AY506768 } \\
\text { AB250626 } \\
\text { AY506778 } \\
\text { AY242051 } \\
\text { AY506756 } \\
\text { AY506755 } \\
\text { AY506754 } \\
\text { AY506798 } \\
\text { AY506781 } \\
\text { AB250630 } \\
\text { JX644883 } \\
\text { AY506797 } \\
\text { KY652919 } \\
\text { JX644885 } \\
\text { AY506786 } \\
\text { AY506785 } \\
\text { AY506790 } \\
\text { AB250636 } \\
\text { JX644886 } \\
\text { AY506784 } \\
\text { AY506758 } \\
\text { AY506762 } \\
\text { AY506760 } \\
\text { AY506783 } \\
\text { AB250639 } \\
\text { AY506796 } \\
\text { AY506780 } \\
\text { AY506791 } \\
\text { AY506801 } \\
\text { AY506801 } \\
\text { JX644884 } \\
\text { AB250641 } \\
\text { JX644888 } \\
\text { JX644889 } \\
\text { AY506799 } \\
\text { AY506802 } \\
\text { AY506759 } \\
\text { AY506792 }\end{array}$ \\
\hline Cariceae & Carex esenbeckii Kunth & Ikeda et al. $20814080(\mathrm{TI})$ & AB643648 \\
\hline Cypereae & $\begin{array}{l}\text { Afroscirpoides dioeca (Kunth) García-Madr. } \\
\text { Cyperus ascocapensis Bauters } \\
\text { Cyperus brevifolius (Rottb.) Hassk. } \\
\text { Cyperus cyperoides (L.) Kuntze } \\
\text { Cyperus involucratus Rottb. } \\
\text { Cyperus isolepis (Nees) Bauters } \\
\text { Cyperus papyrus L. } \\
\text { Cyperus sanguinolentus Vahl } \\
\text { Erioscirpus microstachyus (Boeckeler) Palla } \\
\text { Erioscirpus comosus (Wall.) Palla } \\
\text { Ficinia bergiana Kunth } \\
\text { Ficinia esterhuyseniae Muasya } \\
\text { Ficinia gracilis Schrad. } \\
\text { Ficinia nodosa (Rottb.) Goetgh., Muasya \& D.A.Simpson } \\
\text { Ficinia rigida Levyns } \\
\text { Ficinia spiralis (A.Rich.) Muasya \& de Lange } \\
\text { Ficinia trichodes (Schrad.) Benth. \& Hook.f. } \\
\text { Hellmuthia membranacea (Thunb.) R.W.Haines \& Lye } \\
\text { Isolepis cernua (Vahl) Roem. \& Schult. } \\
\text { Isolepis crassiuscula Hook.f. } \\
\text { Isolepis hystrix (Thunb.) Schrad. } \\
\text { Isolepis levynsiana Muasya \& D.A.Simpson } \\
\text { Isolepis marginata (Thunb.) A.Dietr. } \\
\text { Isolepis setacea (L.) R.Br. } \\
\text { Isolepis venustula Kunth } \\
\text { Scirpoides holoschoenus (L.) Soják } \\
\text { Scirpoides thunbergii (Schrad.) A.Spreng. }\end{array}$ & $\begin{array}{l}\text { Muasya } 3062(\mathrm{BOL}) \\
\text { Muasya } 1009(\mathrm{EA}, \mathrm{K}) \\
\text { Hirahara \& Yano } 18135(\mathrm{OKAY}) \\
\text { Morimoto } 17532(\mathrm{OKAY}) \\
\text { Kew Acc. } 6136603(\mathrm{~K}) \\
\text { Muasya } 1217(\mathrm{~K}) \\
\text { Hepper } 4213(\mathrm{~K}) \\
\text { Komagine \& Masyo } 17655 \text { (OKAY) } \\
\text { Noltie } 2001562(\mathrm{E}) \\
\text { Ikeda et al. } 20814007(\mathrm{TI}) \\
\text { Muasya } 2337(\mathrm{BOL}) \\
\text { Muasya } 2312(\mathrm{BOL}) \\
\text { Faden et al. } 96 / 433(\mathrm{~K}) \\
\text { Wilson } 9455(\mathrm{~K}) \\
\text { Muasya } 2319(\mathrm{~K}) \\
\text { HUG 2003-0699 (GENT) } \\
\text { Muasya } 2328(\mathrm{~K}) \\
\text { Muasya } 3081(\mathrm{BOL}) \\
\text { Muasya } 3073(\mathrm{BOL}) \\
\text { Hirahara \& Hoshino } 19165(\mathrm{OKAY}) \\
\text { Muasya } 2971(\mathrm{BOL}) \\
\text { Muasya } 1151(\mathrm{~K}) \\
\text { Muasya } 2973(\mathrm{BOL}) \\
\text { Ikeda et al. } 20814046(\mathrm{TI}) \\
\text { Muasya } 1189(\mathrm{~K}) \\
\text { HBuG } 2003-1536(\mathrm{GENT}) \\
\text { Muasya } 1205(\mathrm{~K})\end{array}$ & $\begin{array}{l}\text { GU012394 } \\
\text { AB685858 } \\
\text { AB261669 } \\
\text { AB261665 } \\
\text { AY242052 } \\
\text { AB685866 } \\
\text { AY242048 } \\
\text { AB261671 } \\
\text { AB643639 } \\
\text { AB643639 } \\
\text { AB685861 } \\
\text { GU012400 } \\
\text { AB685862 } \\
\text { GU012383 } \\
\text { AB685863 } \\
\text { GU012395 } \\
\text { AB685864 } \\
\text { GU012384 } \\
\text { GU012413 } \\
\text { AB261668 } \\
\text { GU012388 } \\
\text { AB685865 } \\
\text { GU012418 } \\
\text { AB643644 } \\
\text { GU012421 } \\
\text { AB685867 } \\
\text { AB685868 }\end{array}$ \\
\hline Dulichieae & Dulichium arundinaceum (L.) Britton & Waterway 2003.052 (MTMG) & DQ998949 \\
\hline Eleocharideae & $\begin{array}{l}\text { Eleocharis mamillata (H.Lindb.) H.Lindb. } \\
\text { Eleocharis neozelandica C.B.Clarke ex Kirk. } \\
\text { Eleocharis pusilla R.Br. } \\
\text { Eleocharis quinqueflora (Hartmann) O.Schwarz } \\
\text { Eleocharis wichurae Boeckeler }\end{array}$ & $\begin{array}{l}\text { Bures et al. s.n. (BRNU) } \\
\text { Gardner et al. AK284635 (AK) } \\
\text { Gardner et al. AK284890 (AK) } \\
\text { Bures et al. s.n. (BRNU) } \\
\text { Hoshino et al. } 17616 \text { (OKAY) }\end{array}$ & $\begin{array}{l}\text { GU977089 } \\
\text { DQ385566 } \\
\text { DQ385564 } \\
\text { GU977095.1 } \\
\text { AB180715 }\end{array}$ \\
\hline
\end{tabular}


Table 1 (cont.)

\begin{tabular}{|c|c|c|c|}
\hline Tribe & Taxon & Voucher & Accession number \\
\hline \multirow[t]{2}{*}{ Fuireneae } & Actinoscirpus grossus (L.f.) Goetgh. \& D.A.Simpson & Katsuyama et al. 19915 (OKAY) & AB261672 \\
\hline & Schoenoplectus hotarui (Ohwi) Holub & Katayama 17521 (OKAY) & AB180720 \\
\hline Schoeneae & Cladium chinense Nees ex Hook. \& Arn. & H.-K. Choi 2006 s.n. (AJOU) & GQ130342 \\
\hline \multirow[t]{9}{*}{ Scirpeae } & Amphiscirpus nevadensis (S.Watson) Oteng-Yeb. & Helmkamp s.n. (RSA) & AF190618 \\
\hline & Eriophorum angustifolium Honck. & Waterway 2001.018 (MTMG) & DQ998950 \\
\hline & Eriophorum gracile Koch & Hoshino et al. 17382 (OKAY) & AB261684 \\
\hline & Scirpus mitsukurianus Makino & J. Jung 808304 (AJOU) & GQ130354 \\
\hline & Scirpus wichurae Boeckeler & J. Jung 808322 (AJOU) & GQ130357 \\
\hline & Trichophorum alpinum (L.) Pers. & Sato 13260 (OKAY) & AB206270 \\
\hline & Trichophorum dioicum (Y.N.Lee \& Y.C.Oh) J.Jung \& H.K.Choi & J. Jung 804015 (AJOU) & FJ797641 \\
\hline & Trichophorum pumilum (Vahl) Schinz \& Thell. & Ikeda et al. 20814101 (TI) & AB643647 \\
\hline & Trichophorum subcapitatum (Thwaites \& Hook.) D.A.Simpson & Ikeda et al. 10042360 (TI) & AB679909 \\
\hline
\end{tabular}

The aligned matrix yielded 837 characters, of which 419 were parsimony-informative, 116 were variable but parsimony-uninformative and 302 were constant.

Parsimony analysis recovered 16 equally parsimonious trees (Fig. 4), and the ITS phylogeny is similar to results previously published using plastid (e.g., Ghamkar et al. 2007, Muasya et al. 2009) and nuclear (e.g., Yano \& Hoshino 2006, Ghamkar et al. 2007) markers. These strongly supported the Abildgaardieae clade as having two subclades, one comprising Bulbostylis (including Actinoschoenus and Arthrostylis) and the other comprising Fimbristylis. The new taxon was sister to the rest of Fimbristylis in all the 16 trees generated in our study, but there was a lack of bootstrap support for the backbone topology in Fimbristylis in this study as well as previous studies (e.g., Ghamkhar et al. 2007) using trnL-F and ITS sequence data.

On present evidence, the best placement for the new species is in Fimbristylis. However, we were unable to assign the new taxon to any of the sections in Fimbristylis s.l. recognized by previous researchers (e.g., Kern 1974) and further studies are needed to elucidate the precise relationships of the new taxon.

\section{TAXONOMIC TREATMENT}

Fimbristylis fusiformis Wangwasit \& D.A.Simpson, sp. nov. - Fig. 1-3; Map 1

Superficially similar to $F$. pauciflora R.Br. but distinguished by the glumes 6.5-8.5 mm long (vs 2.5-3 mm long in F. pauciflora), nutlets fusiform, fimbriate at apex and base, with 3 longitudinal costae (vs nutlets obovate and glabrous in F. pauciflora). - Type: K. Wangwasit 080927-17 (holo K; iso BK, KKU), Thailand, Ubon Ratchathani, Pha Taem National Park, 27 Sept. 2008.

Etymology. Named after the shape of the nutlets.

Rhizomatous perennial. Culms densely tufted, $12-23 \mathrm{~cm}$ by 0.1-0.4 mm, trigonous, glabrous. Leaves basal; blade narrowly linear, $4-5 \mathrm{~cm}$ by $0.5 \mathrm{~mm}$, obtuse, thickly crescentiform in cross-section, laterally flattened, glabrous; sheath $1-1.5 \mathrm{~cm}$ long, stramineous, sides membranous; ligule absent. Involucral bracts glume-like. Inflorescence a single terminal spikelet. Spikelet linear-cylindric, terete, $1 \mathrm{~cm}$ by $1.2-1.5 \mathrm{~mm}$. Glumes 9 per spikelet, spirally arranged, lower glume glabrous, uppermost strigose, oblong elliptic, $6.5-8.5$ by $1.5-2.3 \mathrm{~mm}$, acuminate, sides membranous, stramineous, reddish brown tinged, keel obtuse, 1-nerved. Stamens 3; anthers 2-2.5 by 0.1-0.2 mm. Stigma branches 3; style persistent; style base rather indistinct, somewhat elongate, gradually widening into the nutlet, scabrous. Nutlets fusiform, trigonous, fimbriate, especially at apex and base, $2.5-3.8$ by $0.5-0.85 \mathrm{~mm}$, apex brown to dark brown, with minute hexagonal epidermal cells, longitudinal costae present.
Distribution - Endemic to eastern Thailand. Mostly seen in Pha Taem National Park but also observed in Phu Chong $\mathrm{Na}$ Yoi National Park, both in Ubon Ratchathani.

Habitat \& Ecology — Open, stony places with scattered tree cover on seasonally wet, sandy soils. Altitude $227 \mathrm{~m}$ (Google Earth 2016).

Conservation status — Least Concern (LC; IUCN 2012). The species occurs in protected areas and is locally abundant in these localities.

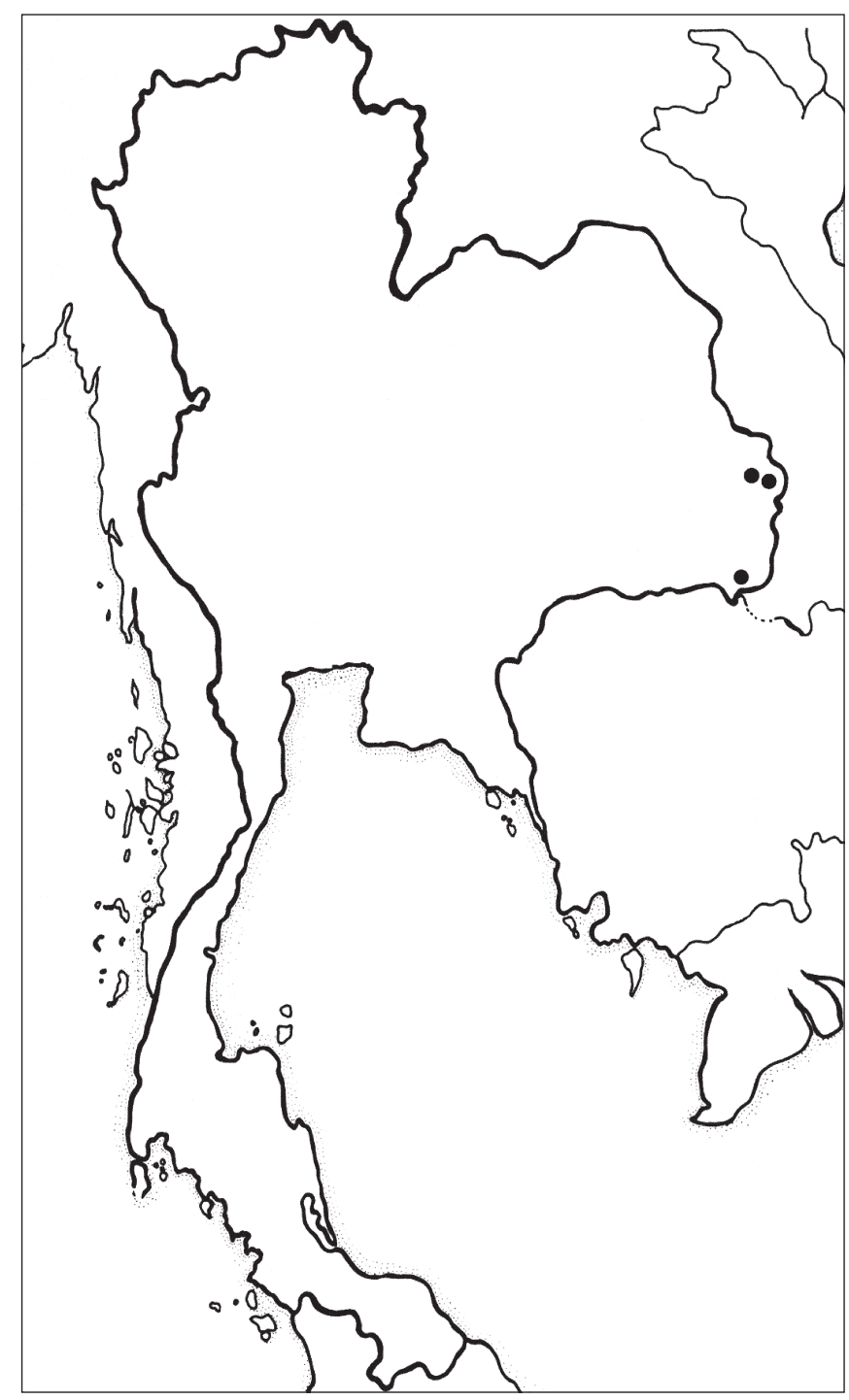

Map 1 Distribution of Fimbristylis fusiformis Wangwasit \& D.A.Simpson. 

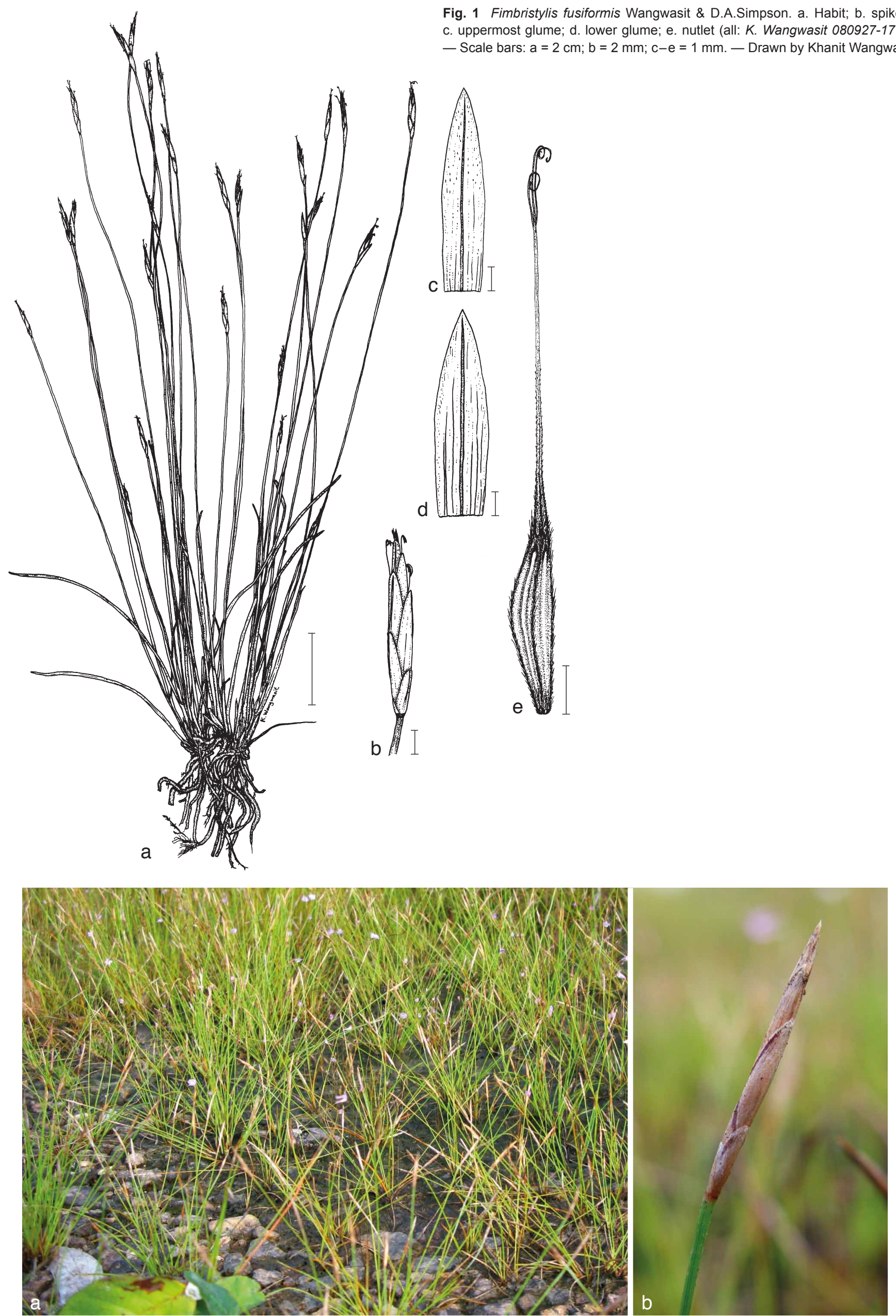

Fig. 2 Fimbristylis fusiformis Wangwasit \& D.A.Simpson. a. Plants in habitat; b. close-up of spikelet. — Photos by D.A. Simpson. 

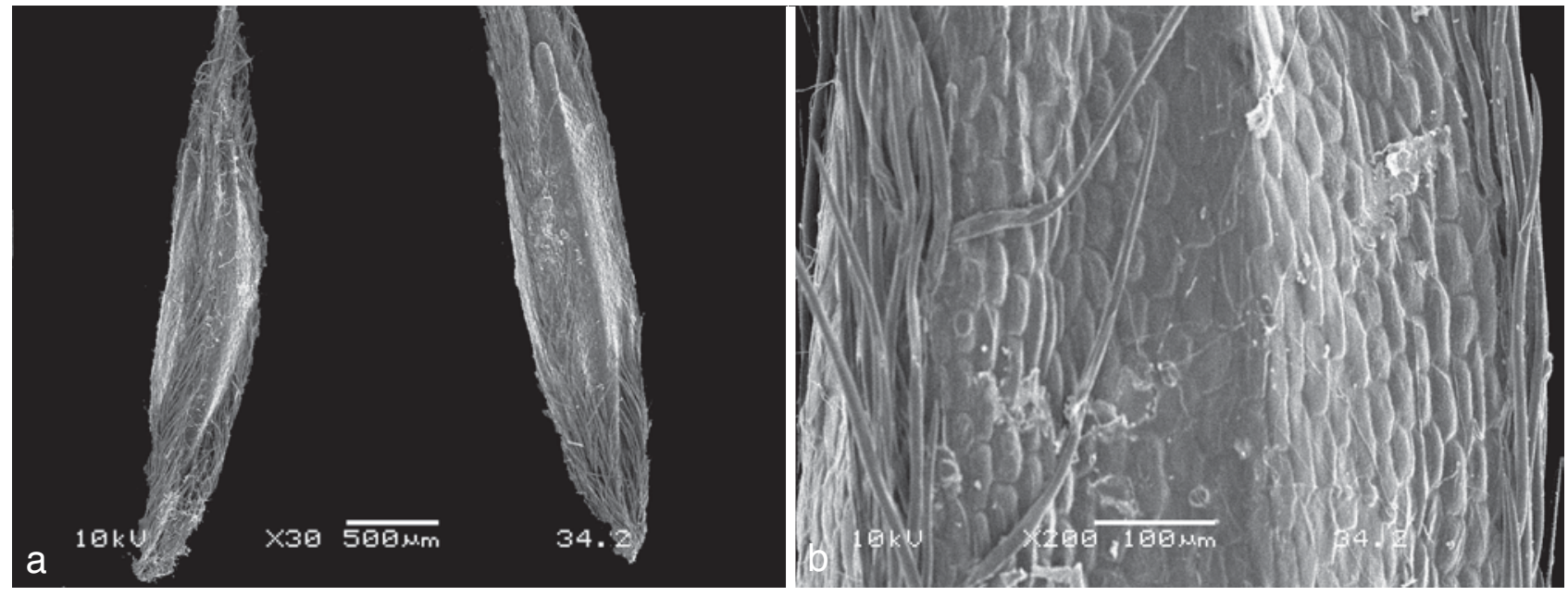

Fig. 3 SEM micrographs of the nutlet of Fimbristylis fusiformis Wangwasit \& D.A.Simpson. a. Whole nutlet; b. nutlet surface (from K. Wangwasit 080927-17, K).

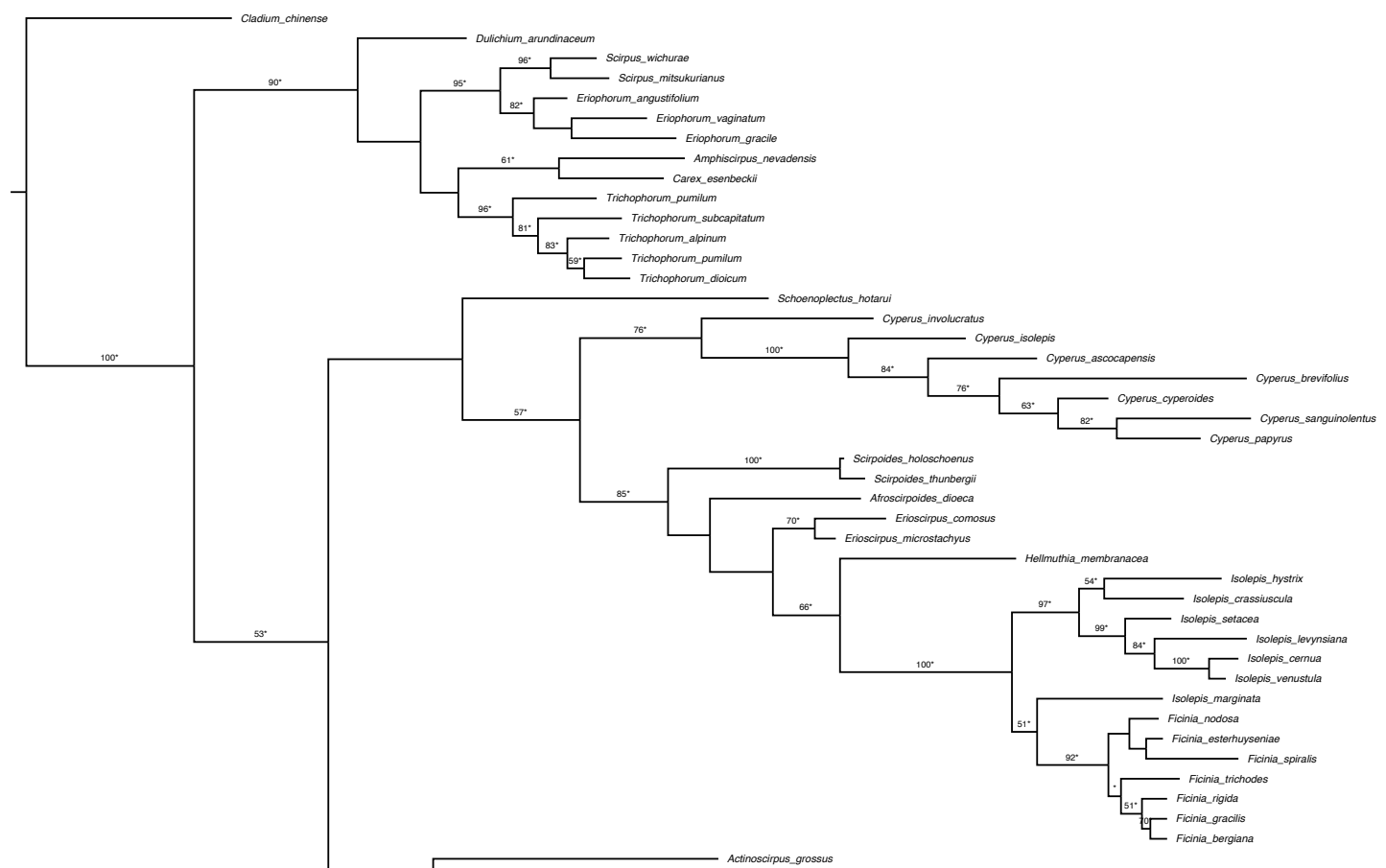

Fig. 4 One of 16 maximum parsimony trees, showing the position of Fimbristylis fusiformis in the Abildgaardieae clade (highlighted in the grey box). Nodes observed in the strict consensus tree are marked with an asterisk $\left(^{*}\right)$ and bootstrap support values greater than $50 \%$ are indicated above the branches before the nodes.

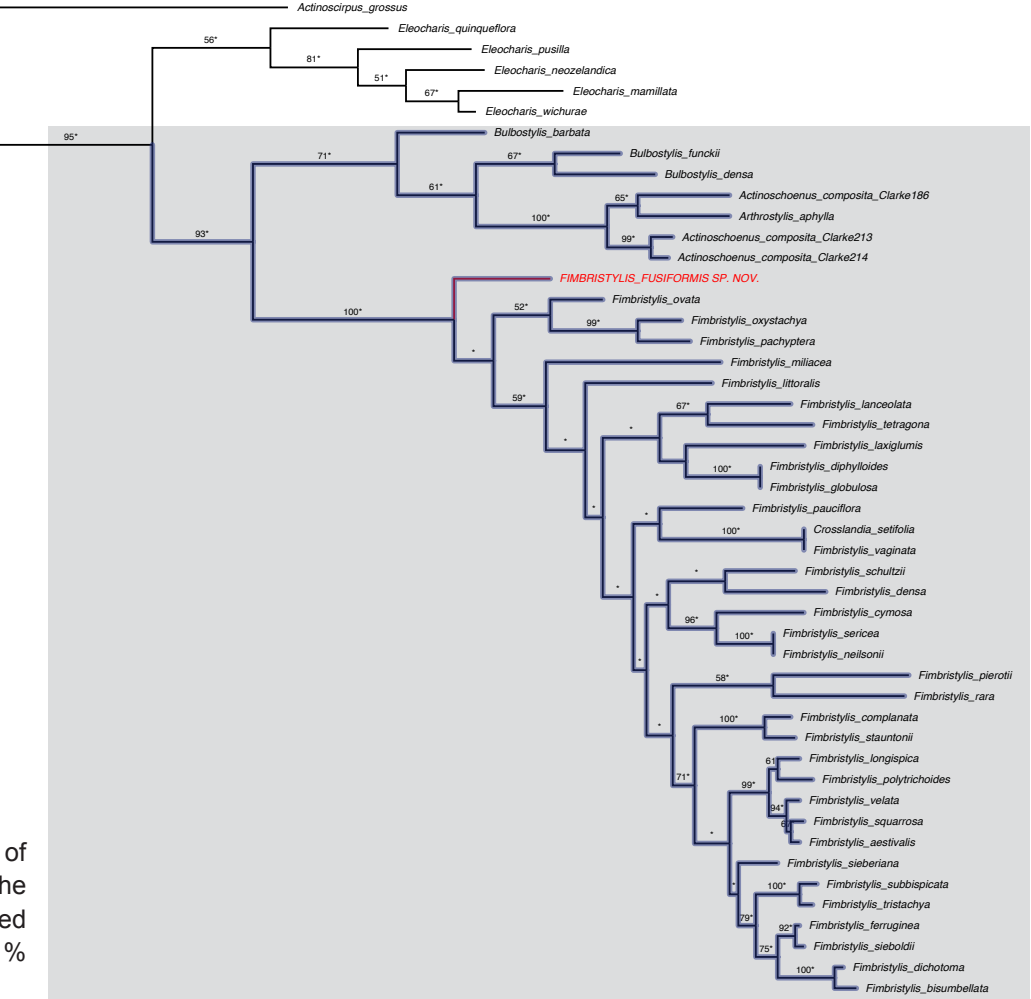


Additional specimens examined. THAILAND, Ubon Ratchathani: Khong Chiam, Pha Taem National Park, Khua Nangnee Falls (N15²4'11" E105³1'02"), Pooma, De Wilde \& Duyfjes 2270 (BKF); Sribunreung District, 27 Sept. 2008, K. Wangwasit 080927-6 (KKU).

Note - Fimbristylis fusiformis is superficially similar to F. pauciflora, in having an inflorescence with a single terminal linear-cylindric spikelet bearing a glume-like bract. We interpret these similarities to be due to morphological convergence, as the two taxa are not sister in our phylogeny.

Acknowledgements We acknowledge the curators of BK, BKF, K, KKU and QBG for allowing access to their collections and for their kind assistance. The first author wishes to thank Mrs Wilailux Zumstein who prepared silica dried leaves for DNA extraction and Dr Khanit Wangwasit for collecting plant material and for preparing the line drawing. The annual government statement Year 2011 of Mahasarakham University is thanked for financial support.

\section{REFERENCES}

Edgar RC. 2004. MUSCLE: multiple sequence alignment with high accuracy and high throughput. Nucleic Acids Research 32: 1792-1797.

Ghamkhar K, Marchant AD, Wilson KL, et al. 2007. Phylogeny of Abildgaardieae (Cyperaceae) inferred from ITS and trnL-F data. Aliso 23: 149-164.

Goetghebeur P. 1998. Cyperaceae. In: Kubitzki K (ed), The families and genera of vascular plants 4: 141-189. Springer-Verlag, Berlin, Heidelberg Google Earth v. 7.1.7.2600. 2016. N15²4'11.0" E105³1'02.0", elevation 227 m. http://www.google.com/earth/index.html [Viewed 1 Sept. 2016].

Govaerts R, Simpson DA, Bruhl JJ, et al. 2007. World checklist of Cyperaceae. Kew Publishing.

Hall TA. 1999. BioEdit: a user-friendly biological sequence alignment editor and analysis program for Windows 95/98/NT. Nucleic Acids Symposium Series 41: 95-98.

IUCN. 2012. IUCN Red List categories and criteria: Version 3.1. Second edition. IUCN, Gland, Switzerland and Cambridge, UK.

Kern JH. 1974. Cyperaceae 1. In: Van Steenis CGGJ (ed), Flora Malesiana, Series I, 7: 435-753. Noordhoff International Publishing, Leiden.

Muasya AM, Simpson DA, Verboom GA, et al. 2009. Phylogeny of Cyperaceae based on DNA sequence data: current progress and future prospects. Botanical Review 75: 2-21.

Muasya AM, Viljoen J-A, Dludlu MN, et al. 2014. Phylogenetic position of Cyperus clandestinus (Cypereae, Cyperaceae) clarified by morphological and molecular evidence. Nordic Journal of Botany 32: 106-114

Simpson DA, Furness CA, Hodkinson TR, et al. 2003. Phylogenetic relationships in Cyperaceae subfamily Mapanioideae inferred from pollen and plastid DNA sequence data. American Journal of Botany 90: 1071-1086.

Simpson DA, Koyama T. 1998. Cyperaceae. In: Santisuk T, Larsen K (eds), Flora of Thailand 6, 4: 247-485. Diamond Printing, Bangkok, Thailand.

Staden R. 1996. The Staden sequence analysis package. Molecular Biotechnology 5: 233-241.

Swofford DL. 2002. PAUP*: phylogenetic analysis using parsimony ("and other methods), v. 4. Sinauer Associates, Inc., Sunderland, Massachusetts, USA.

Yano O, Hoshino T. 2006. Molecular phylogeny and chromosomal evolution of Japanese Schoenoplectus (Cyperaceae), based on ITS and ETS $1 f$ sequences. Acta Phytotaxonomica et Geobotanica 56: 183-195.

Yano O, Ikeda H, Watson MF, et al. 2012. Phylogenetic position of the Himalayan genus Erioscirpus (Cyperaceae) inferred from DNA sequence data. Botanical Journal of the Linnean Society 170: 1-11. 